\title{
Vitamin D and diabetes: the devil is in the D-tails
}

\author{
C. Mathieu
}

Received: 30 March 2010 /Accepted: 1 April 2010/Published online: 21 May 2010

(C) Springer-Verlag 2010

\begin{tabular}{|c|c|}
\hline Abbreviations & \\
\hline CYP27B1 & $\begin{array}{l}\text { Cytochrome P450, family } 27 \text {, } \\
\text { subfamily B, polypeptide } 1\end{array}$ \\
\hline DIPP & Diabetes Prediction and Prevention \\
\hline $25-(\mathrm{OH}) \mathrm{D}$ & 25-Hydroxyvitamin D \\
\hline $1,25-(\mathrm{OH}) 2 \mathrm{D}$ & 1,25-Dihydroxyvitamin D \\
\hline PTH & Parathyroid hormone \\
\hline VDR & Vitamin D receptor \\
\hline
\end{tabular}

Is vitamin $\mathrm{D}$ important in diabetes? In the current issue of Diabetologia, Marjamäki et al. report data from the Diabetes Prediction and Prevention Study (DIPP), in which the dietary habits of Finnish women were meticulously examined and linked to the onset of autoimmunity in their (genetically at risk) offspring [1]. The straightforward answer from this impressive study is that there was no correlation between the amount of vitamin D consumed by the mother and the appearance of islet autoantibodies in the blood of their offspring in the first year of life. These data clarify one important issue, but do not resolve the existing confusion about vitamin $\mathrm{D}$ and diabetes. Some experts still claim an important role for this molecule, and lay websites advocate vitamin D supplementation in high doses to prevent type 1 and type 2 diabetes. The devil is in the detail....

Vitamin D, strictly defined, is not a vitamin as it is not a substance that needs to be present in foods in small quantities in order to avoid pathology, as with vitamin $\mathrm{C}$ and scurvy. Few

C. Mathieu $(\bowtie)$

Department of Endocrinologoy, UZ Gasthuisberg,

Katholieke Universiteit Leuven,

3000 Leuven, Belgium

e-mail: chantal.mathieu@med.kuleuven.be

natural foods contain useful quantities of vitamin D. Cod liver oil and oily fish are considered to be rich sources, whereas butter, cream and egg yolk contain only small amounts. Milk, whether from humans or cows, is a poor source of vitamin D [2]. Most vitamin $\mathrm{D}$, in fact, comes from our own skin, in which we convert 7-dihydrocholesterol (by photolytic cleavage) into pre-vitamin $\mathrm{D}_{3}$, which is subsequently converted by spontaneous thermal isomerisation into vitamin $\mathrm{D}_{3}$ [2] (see Fig. 1). As only certain wavelengths of UV light (270-300 nm) can achieve this effect, the sun needs to rise above a certain angle in the sky (equivalent to a UV index of 3) before this condition is achieved. This only occurs for a few months in the year in areas with a northerly location such as Finland, and sitting for hours in the sun (if you are lucky enough to see it...) during the month of February in Belgium or the UK would make no vitamin D at all. Unfortunately, the wavelengths necessary for making vitamin $\mathrm{D}$ in our skin are exactly the same as those involved in skin ageing and skin cancer. So, what is our safest source of vitamin D? Reasonable sun exposure is one source, but supplements will also be necessary.

What, in all events, is the case for supplementing vitamin D? Here we build mainly on epidemiological data and studies performed in vitro or in animal models. Several reports exist on worsening of glycaemic control in type 2 diabetic patients with 


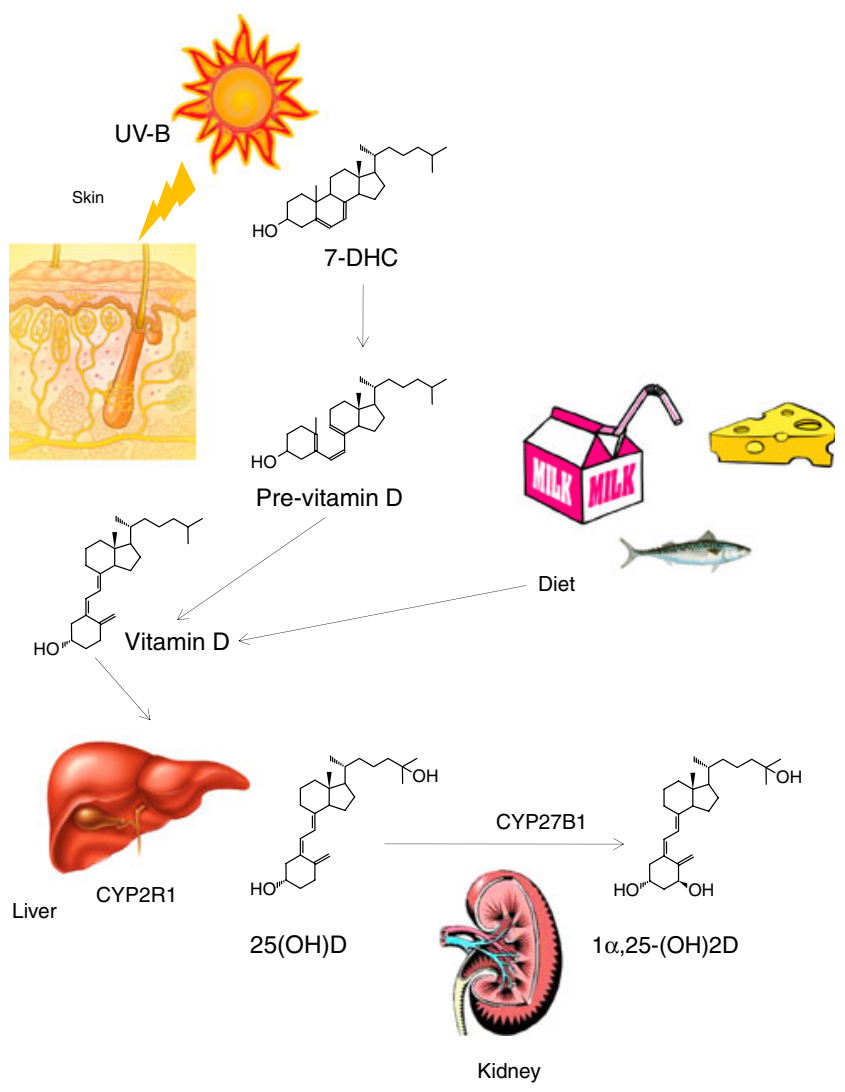

Fig. 1 Metabolism of vitamin D. Synthesis of vitamin $D_{3}$ occurs in the skin where 7-dehydrocholesterol (7-DHC) is converted to previtamin D in response to ultraviolet light (UV) exposure. Vitamin D, obtained from pre-vitamin $\mathrm{D}$ in the skin or by intestinal absorption of dietary components, binds to vitamin D-binding protein in the circulation and is transported to the liver. Here, vitamin $\mathrm{D}$ is hydroxylated by liver 25-hydroxylase (CYP2R1). The resulting 25 $(\mathrm{OH}) \mathrm{D} 3$ is then hydroxylated in the kidney by $1-\alpha$-hydroxylase (CYP27B1), generating the active hormone 1,25-(OH)2D

overt vitamin D deficiency (in the range of rickets in children and osteomalacia in adults, $<12.5-37.5 \mathrm{mmol} / 1[<5-15 \mathrm{ng} / \mathrm{ml}]$ ) and on a link between developing rickets in the first year of life and subsequent risk of type 1 diabetes [3]. These epidemiological human data are strengthened by in vitro data indicating that rachitic animals have beta cells that function badly, and go on to develop glucose intolerance. In the NOD mouse, a model for human type 1 diabetes, severe vitamin $\mathrm{D}$ deficiency doubles the rate of diabetes development in later life. Thus, one statement concerning vitamin $\mathrm{D}$ and diabetes can be made with a reasonable degree of certainty: avoid severe vitamin $\mathrm{D}$ deficiency.

What is the role of vitamin D supplementation above the threshold of vitamin D deficiency? Here the D-details become even more important. These data come from epidemiology and from anecdotally reported interventions in small numbers of individuals that have used very different amounts of vitamin D or its metabolites. Epidemiological studies, such as that by Marjamäki et al., build their claims on dietary questionnaires, rather than actual plasma levels of vitamin $\mathrm{D}$ before reaching conclusions about possible links between 'vitamin D and diabetes'. Try remembering what you ate when you were pregnant - not very efficient. Another point to consider is publication bias, such that studies demonstrating the existence of a correlation are more likely to be published. One striking conclusion from the present study is the fact that recommendations on dietary intakes and supplements are very hard to implement, even in a special population such as pregnant women. In fact, only $15 \%$ stated that they took more than $10 \mu \mathrm{g}$ (400 international units [IU]) of vitamin D per day, and only $30 \%$ of women actually took supplements [1].

Could blood sampling for vitamin D levels help in epidemiological studies? It would surely give more trustworthy information, but what then should be measured? In order for vitamin D to become 'active' it needs to undergo two subsequent hydroxylations: one in the liver at the 25 position and one in the kidney at the 1 position resulting in, respectively, 25-hydroxyvitamin D (25-[OH]D) and 1,25-dihydroxyvitamin D (1,25-[OH]2D) [4] (see Fig. 1). Measuring 1,25-(OH)2D has the advantage of measuring 'the real thing', the active product, that will interact with the vitamin D receptor (VDR), a nuclear receptor responsible for the effects of vitamin $\mathrm{D}$ in target organs, such as beta cells and immune cells. However, this product is present in $\mathrm{pg} / \mathrm{pmol}$ and is therefore very hard to measure and ideally needs sophisticated techniques such as liquid chromatography-tandem mass spectrometry (LCMSMS). Moreover, the titres of this product are highly variable and depend on the activity of cytochrome P450, family 27, subfamily B, polypeptide 1 (CYP27B1), the enzyme in the kidneys responsible for the 1-hydroxylation. This enzyme is under the control of parathyroid hormone (PTH) and will vary according to the global vitamin D status, and also bone needs. Furthermore, there is active discussion as to whether 'non-bone' tissues, such as the beta cells or immune system, really depend on circulating $1,25-(\mathrm{OH}) 2 \mathrm{D}$ at all, because they can make it themselves. Many tissues do indeed possess CYP27B1 and will be able to activate vitamin $\mathrm{D}$ in a non-PTH-dependent manner. For instance, in monocytes and macrophages, CYP27 becomes activated in the presence of inflammatory agents such as cytokines, and on Toll-like receptor activation, making these non-bone tissues independent of circulating 1,25-(OH)2D [5]. Is 25-(OH)D therefore a better measure of vitamin D status? It does indeed have several advantages: it is present in the millimoles per litre range, which makes it easier to measure; and it is a direct reflection of vitamin D reserves in the body, as the enzymes responsible for 25-hydroxylation in liver (CYP27A1 and at least three others) will convert all vitamin $\mathrm{D}$ that comes into the body into $25-(\mathrm{OH}) \mathrm{D}$ in a non-rate-limiting way. These levels are quite stable over several days or weeks and fluctuate typically with vitamin D intake and UVB exposure (seasonal variation). 
And now we come to the genetic D-details: polymorphisms exist for almost all enzymes involved in vitamin D metabolism (also in CYP24, the 24hydroxylase responsible for inactivating 1,25-[OH]2D), as well as for the carrier protein [vitamin D binding protein, DBP] and the VDR). Thus, a similar level of 25-(OH)D may reflect a very different tissue exposure to $1,25-(\mathrm{OH}) 2 \mathrm{D}$ locally [6]. This enables us to make another statement about vitamin $\mathrm{D}$ and diabetes: global vitamin D load can be measured as $25-(\mathrm{OH}) \mathrm{D}$ in plasma, but genetic phenotyping will be crucial in determining the actual 'vitamin D' exposure in the target tissues. This means that different individuals may have different requirements for vitamin D to maintain 'non-bone' health.

What about intervention trials using high doses of vitamin D or metabolites in diabetes? Here we are limited to small interventions and animal studies. In type 2 diabetes very high doses of 25-(OH)D have been administered, but the data are confusing, whereas in type 1 diabetes 1,25 $(\mathrm{OH}) 2 \mathrm{D}$ has mainly been given, with little or no effect on C-peptide preservation in newly diagnosed type 1 patients $[7,8]$. What about NOD mice? The D-details once again matter. In NOD mice, only very high doses of 1,25-(OH)2D administered over many weeks early in life are able to prevent diabetes. Supplementing with small doses (up to $1000 \mathrm{IU})$ of regular vitamin D does not prevent diabetes in NOD mice. Once autoimmunity was present, neither regular vitamin $\mathrm{D}$ nor high doses of $1,25-(\mathrm{OH}) 2 \mathrm{D}$ were able to reverse the disease. In this situation, only analogues of 1,25-(OH)2D (best when combined with regular immunosuppressants over the short term) could affect disease progression [3]. These analogues are chemically altered $1,25-(\mathrm{OH}) 2 \mathrm{D}$ molecules with preserved action on tissues such as beta cells and immune cells, and less effect on bone and calcium metabolism [9]. Until now, only one analogue has been marketed for treatment of an autoimmune disease: calcipotriol (Daivonex) for topical application in psoriasis. One further statement can be made with certainty about vitamin $\mathrm{D}$ and diabetes: in animal models (mostly the NOD mouse) high doses of 1,25-(OH)2D reduce the incidence of diabetes in primary prevention studies.

So, what do we need before we can finally make a definitive statement about vitamin $\mathrm{D}$ and diabetes in humans? We need studies, more specifically intervention studies, randomised and placebo controlled. Why don't we have them yet? Besides the fact that regular vitamin D is cheap and trials will therefore need to be investigatorinitiated, the major problem is that the effect sizes may be rather small, requiring big populations - a huge and expensive undertaking. Studies may be more feasible in type 2 diabetes, or in glucose-impaired individuals, provided that sophisticated tools for measuring beta cell function in vivo are used (e.g. clamps). In type 1 diabetes, the issue is more difficult, because here the NOD mouse shows us that primary prevention is the best way to go, which implies intervening before disease - and, preferably, before autoimmunity - is present. Two paths can be followed. One would be a massive primary prevention trial on a population level: for example, all neonates east of the Mississippi get an extra amount of vitamin D per day during their first year of life, those west of the Mississippi do not, and we observe the development of autoimmunity and type 1 diabetes over $5-10$ years. How much vitamin D? How many units? Here safety has to prevail as vitamin $\mathrm{D}$, being fat soluble, can cause hypercalcaemia, hypercalciuria and kidney stones (despite what some vitamin D gurus would wish us to believe). Giving an extra 1,000 IU above the recommended daily amount could be defended, but time is pressing as more and more individuals are supplementing themselves and their children on the basis of messages in the lay press. The second, more scientific, path would be to screen for genetically at-risk neonates, allow a higher supplement (e.g. $2,000 \mathrm{IU}$ or more) and observe autoimmunity or type 1 diabetes onset over the following years. In such a study, data on genetic markers for vitamin D metabolism should be collected, thus allowing for differences in effect due to the presence of certain polymorphisms, and identifying individual phenotypes in which larger or smaller amounts of vitamin D might be needed. What about intervention when autoimmunity or even type 1 diabetes is already present? There I still believe we need a potent analogue, without calcaemic effects, before we can embark on any intervention.

In conclusion, the paper in this issue of Diabetologia does not alter the story on vitamin $\mathrm{D}$ and diabetes, but highlights the difficulties in 'getting it right'. Making conclusions on the vitamin D status in utero of a fetus on the basis of what the mother recalls eating in pregnancy is difficult and this 'negative' study is one more in the list of epidemiological studies on the topic. What we really need are carefully designed prospective placebo-controlled and randomised trials to provide definitive answers as to the sense or non-sense of vitamin D supplementation in individuals affected by, or at risk of, either form of diabetes.

Duality of interest The author declares that there is no duality of interest associated with this manuscript.

\section{References}

1. Marjamäki L, Niinistö S, Kenward MG et al. (2010) Maternal intake of vitamin D during pregnancy and risk of advanced beta 
cell autoimmunity and type 1 diabetes in offspring. Diabetologia. doi:10.1007/s00125-010-1734-8

2. Holick MF (1997) Photobiology of vitamin D. In: Feldman D, Glorieux FH, Pike J (eds) vitamin D. Academic, San Diego, pp 33-39

3. Mathieu C, Gysemans C, Giulietti A, Bouillon R (2005) Vitamin D and diabetes. Diabetologia 48:1247-1257

4. Heaney RP, Horst RL, Cullen DM, Armas LA (2009) Vitamin D3 distribution and status in the body. J Am Coll Nutr 28:252256

5. van Etten E, Stoffels K, Gysemans C, Mathieu C, Overbergh L (2008) Regulation of vitamin D homeostasis: implications for the immune system. Nutr Rev 66:S125-S134
6. Smolders J, Peelen E, Thewissen M et al (2009) The relevance of vitamin $\mathrm{D}$ receptor gene polymorphisms for vitamin $\mathrm{D}$ research in multiple sclerosis. Autoimmun Rev 8:621-626

7. Palomer X, González-Clemente JM, Blanco-Vaca F, Mauricio D (2008) Role of vitamin D in the pathogenesis of type 2 diabetes mellitus. Diab Obes Metab 10:185-197

8. Pitocco D, Crinò A, Di Stasio E, IMDIAB Group et al (2006) The effects of calcitriol and nicotinamide on residual pancreatic betacell function in patients with recent-onset type 1 diabetes (IMDIAB XI). Diabet Med 23:920-923

9. Bouillon R, Verlinden L, Eelen G et al (2005) Mechanisms for the selective action of vitamin D analogs. J Steroid Biochem Mol Biol 97:21-30 